\title{
Dietary patterns, plasma vitamins and Trans fatty acids are associated with peripheral artery disease
}

\author{
Mohsen Mazidi ${ }^{1,2 *}$, Nathan D. Wong ${ }^{3}$, Niki Katsiki ${ }^{4}$, Dimitri P. Mikhailidis ${ }^{5}$ and Maciej Banach ${ }^{6,7}$
}

\begin{abstract}
Background: To investigate the association between dietary patterns (DP), plasma vitamins and trans fatty acids (TFAs) with the likelihood of peripheral artery disease (PAD).

Methods: National Health and Nutrition Examination Survey (NHANES) data for the years 1999-2002 were used. PAD was diagnosed by ankle brachial index assessment. Plasma concentrations of vitamins were measured using high performance liquid chromatography. Vitamin D levels were measured by radioimmunoassay. Analysis of covariance, principal components analysis (PCA) and adjusted logistic regression were applied, accounting for the survey design and sample weights.

Results: Of the 4864 eligible participants, 2482 (51.0\%) were men and 269 (5.5\%) had prevalent PAD. PCA uncovered three DPs which accounted for $56.8 \%$ of the variance in dietary nutrients consumption including DP1 (fatty acids and cholesterol), DP2 (minerals, vitamins and fiber), and DP3 (polyunsaturated fatty acids [PUFA]). PAD patients had a significantly higher serum concentrations of trans 9-octadecenoic acid and trans 9, trans 12-octadienoic acid as well as lower plasma levels of vitamin $\mathrm{D}$, retinol, retinyl stearate and retinyl palmitate ( $p<0.001$ for all comparisons). In models adjusted for age, race, diabetes, cholesterol, hypertension, smoking and energy intake, individuals in the highest quartile of the DP1 had higher odds for PAD compared with those in the lowest quartile [(odds ratio (OR): 6.43, 95\% confidence interval (CI): 2.00-20.63 $p<0.001$ ], while those in the highest quartile of DP2 and DP3 had lower odds of PAD relative to those in the lowest quartile (OR:0.28, OR:0.44, respectively; $p<0.001$ for both comparisons).

Conclusion: We found that quality of diet, plasma vitamins and TFAs are associated with the likelihood of PAD. If confirmed in prospective studies, the possibility that dietary factors, plasma vitamins and TFAs might be valuable for preventing or delaying the clinical progression of PAD, should be investigated in intervention trials.
\end{abstract}

Keywords: Peripheral artery disease, Dietary patterns, Vitamins, Trans fatty acids

\section{Background}

Peripheral artery disease (PAD) is a debilitating, chronic disease caused by the development of atherosclerotic plaques in the arteries of the lower extremities. In contrast to other cardiovascular diseases, little is known about the

\footnotetext{
* Correspondence: moshen@genetics.ac.cn

${ }^{1}$ Key State Laboratory of Molecular Developmental Biology, Institute of Genetics and Developmental Biology, Chinese Academy of Sciences, Chaoyang, Beijing, China

${ }^{2}$ Institute of Genetics and Developmental Biology. International College, University of Chinese Academy of Science (IC-UCAS), West Beichen Road, Chaoyang, China

Full list of author information is available at the end of the article
}

role of diet in PAD [1]. Smoking, hypercholesterolemia, hypertension, diabetes mellitus and chronic renal failure are well-recognized risk factors for PAD besides age [2]. However, the complex relationships among these factors make it difficult to disentangle their respective contributions.

In humans, there are a few studies on PAD limited to specific nutrients, often of modest sample size [3-6]. In this regard, vitamin $C$ has been recognised to have an anti-atherogenic effect based on the association between PAD and subclinical vitamin C deficiency [7]. Although both antioxidant and anti-inflammatory properties might 
explain such an effect, the possibility also exists that vitamin $\mathrm{C}$ deficiency is a marker of unhealthy diet or lifestyle instead of being an independent risk factor. Also omega-3 fatty acids, which are effective in both prevention and treatment of coronary artery disease, have been suggested to protect from PAD, but omega-3 fatty acid supplementation failed to improve hemodynamic or clinical outcomes in PAD patients [8]. Available studies have mostly focused on a single nutrient or food/food group in relation with PAD [3-6]. These common approaches have methodological and conceptual limitations $[9,10]$, in the sense that they can detect the effects of only a single nutrient or food on overall health but would not explain the interactions among nutrients and foods [11]. In addition, such studies do not provide tangible practical dietary advice, as nobody normally consumes just a single food or nutrient $[10,12]$.

Dietary pattern (DP) analysis has emerged as an alternative approach to nutritional epidemiology [13, 14]. In this approach, statistical methods are used to examine the pattern of intake of multiple foods or nutrients and derive single-exposure variables or DP. Such DP may provide an improved and more generalizable insight into diet-disease relations (9). The use of the DP approach could facilitate the development of public health recommendations that are clearer and more convenient to follow [15]. As people eat meals consisting of a variety of food items and complex combinations of nutrients, the traditional approach (focusing on the intake of a single micronutrient/macronutrient) does not necessarily take into account the cumulative inter-correlations and interactions between foods and nutrients $[9,16]$.

However, it is still largely unknown how DP may be associated with vascular disease. The purposes of this study were: (a) to identify the DP for a national sample of American adults, (b) to compare the adherence to each DP between individuals with or without PAD (at the same time correcting for main clinical and demographical confounders) for each gender, (c) to determine whether a DP analysis can have an impact on the likelihood of PAD (at the same time correcting for main clinical and demographical confounders) stratified by gender, and, (d) to determine the associations between plasma vitamins and trans fatty acids (TFAs) with PAD.

\section{Methods}

\section{Population characteristics}

National Health and Nutrition Examination Surveys (NHANES) are ongoing repeated cross sectional surveys in non-institutionalized adults conducted by the US National Center for Health Statistics (NCHS) [17]. The National Center for Health Statistics Research Ethics Review Board approved the NHANES protocol and consent was obtained from all participants [17]. Data collection on demographic information occurs through in-home administered questionnaires, while anthropometrical, inflammation and biochemistry data are collected by trained subjects using mobile exam units. More detailed information is available elsewhere [17, 18]. Dietary intake was assessed via 24-h recall obtained by a trained interviewer during the mobile examination center visit with the use of a computer assisted dietary interview system with standardized probes, i.e. the United States department of Agriculture Automated Multiple-Pass Method (AMPM) [19-21]. AMPM is designed to enhance complete and accurate data collection while reducing respondent burden [20,22, 23].

A blood specimen was drawn from the participant's antecubital vein according to a standardized protocol. Fasting blood glucose (FBG) was measured in plasma by a hexokinase method using a Roche/Hitachi 911 Analyzer (DiaSorin, Stillwater, MN, USA) and Roche Modular P Chemistry Analyzer (DiaSorin, Stillwater, MN, USA). Complete laboratory procedures for collection, storage, calibration and quality control of blood samples for determination of high sensitivity C-reactive protein (hsCRP) concentrations are also available elsewhere $[18,24]$. Insulin was measured using an enzymelinked immunosorbent assay immunoassay (Mercodia, Uppsala, Sweden) [25].

The current study was based on the analysis of data for two 2-year NHANES survey cycles: 1999-2002. The ankle brachial index $(\mathrm{ABI})$ assessment and the lower extremity disease examination were limited to those adults who were $\geq 40$ years (https://www.cdc.gov/nchs/index.htm). More detailed information on the NHANES protocol is available elsewhere [17]. All methods were carried out in accordance with relevant guidelines and regulations $[18$, 25-27]. All experimental protocols were approved by the NCHS [18, 25-27]. Informed consent was obtained from all participants, and the NCHS research ethics review board approved the protocol.

\section{Plasma TFAs and vitamins concentration}

Plasma TFAs were measured as part of the NHANES protocol assessing the total (free and esterified) content of selected TFAs in the plasma and providing results in concentration $(\mu \mathrm{M})$ [28]. In this method, fatty acids in the plasma are converted into free fatty acids by subsequent acidic and alkaline hydrolysis. Free fatty acids are extracted using liquid-liquid extraction and derivatized with pentafluorobenzylbromide (PFB-Br) [28]. The derivatized fatty acids are separated by capillary gas chromatography and detected by mass spectrometry using negative chemical ionization. The fatty acids are identified based on their chromatographic retention time and the specific mass to charge ratio of the ion formed in the ion source. Retention times are then compared against those obtained with known standards [29, 30]. 
Quantitation is performed with standard solution using stable isotope-labelled fatty acids as internal standards. To calculate TFAs as percent of total fatty acids, 29 fatty acids were determined with this measurement procedure. These fatty acids comprise $95 \%$ of all fatty acids present in the plasma [30]. This method determines the following four TFAs: trans-9-hexadecenoic acid (palmitelaidic acid, C16:1n-7 t), trans-9-octadecenoic acid (elaidic acid, C18:1n-9 t), trans-11-octadecenoic acid (vaccenic acid, C18:1n-7 t-), and trans-9, trans-12octadecadienoic acid (linolelaidic acid, C18:2n-6 t, $9 \mathrm{t}$ ) [28]. More detailed information on the NHANES protocol is available in the NHANES manual (https://www.cdc.gov/ nchs/index.htm). Plasma concentrations of vitamins A (retinol) and two retinyl esters, were also measured using high performance liquid chromatography with photodiode array detection as previously detailed [17]. Total plasma 25(hydroxy)vitamin D was measured at the National Center for Environmental Health, CDC, Atlanta, GA, USA using a radioimmunoassay (RIA) kit (DiaSorin, Stillwater, $M N$, USA). The sensitivity of this assay has been shown to be $1.5 \mathrm{ng} / \mathrm{ml}$ and the coefficient of variance (CV) was 7\% [31].

\section{PAD assessment}

ABI was calculated for each participant as follows: with the participant in the supine position, trained health staff used an 8.1-MHz Doppler probe to perform the examination following a standard operation protocol. The ABI was calculated by dividing the ankle mean systolic blood pressure by brachial mean systolic blood pressure in the same side. The presence of PAD was defined as an ABI $<0.9$ in either side [32]. We excluded subjects $(n=40)$ with extremely high ABI (>1.4).

\section{Statistical analysis}

We conducted the analyses according to the guidelines by the Centers for Disease Control and Prevention (CDC) for analysis of complex NHANES datasets, accounting for the masked variance and using the proposed weighting methodology [23, 33]. Principal components analysis (PCA) was applied in order to identify DP [21, 34]. Briefly, PCA with orthogonal transformation (varimax procedure) was performed to derive nutrient patterns based on the nutrients. DPs were retained for further analysis based on their natural interpretation and eigenvalues on the Scree test [34, 35]. We computed the score for each DP by summing up intakes of nutrients weighted by their factor loadings [34, 35]. Each participant received a score for each identified DP. As simple linear dose-response relationships are unlikely to be found in nutritional epidemiology [21, 34, 36]. Analysis of covariance (ANCOVA) was applied to determine the adjusted mean of the nutrients across the quartile of the DPs and also the adjusted mean of each
DP score for individuals with and without PAD stratified by gender. Adjusted logistic and linear (500 bootstrap replications) regression was used to determine the odds of PAD across the each PD and severity of PAD and adhesion to each DPs. All tests were two sided, and $p<$ 0.05 was the level of significance.

\section{Results}

A total of 4864 participants were eligible for inclusion in the current analysis. Of these, 269 (5.5\%) had PAD. The characteristics of participants overall and by PAD presence are summarised in Additional file 1: Table S1. Overall 2482 (51.0\%) participants were men and 2382 (49.0\%) were women, with no significant difference by PAD status $(p=0.423)$. PAD patients, compared with those without PAD, comprised more of non-Hispanic Whites (57.2 vs. 52.4\%), and non-Hispanic Blacks (23.4 vs. $17.6 \%$ ), and less of Mexican-Americans (15.2 vs. $22.6 \%$ ), other Hispanic (3.7 vs. $4.5 \%$ ), or other ethnicities (0.4 vs. $2.9 \%)(p<0.001$ for differences in the distribution of ethnicity by PAD presence).

The mean age was 59.6 years overall, and it was higher in participants with PAD than in those without (70.1 vs. 58.8 years, $p<0.001)$. Furthermore, PAD patients had higher body mass index $(p<0.001)$ as well as higher serum concentrations of hsCRP, triglyceride (TG), FBG, insulin and $\mathrm{HbA} 1 \mathrm{c}(p<0.001$ for all comparisons). Overall, only $0.8 \%$ of the study population $(n=40)$ had extremely high ABI $(>1.4)$ and were not included in our analyses.

By applying PCA, we found three DP which could explain $56.8 \%$ of the variance of the dietary nutrients consumption. Additional file 2: Table S2 illustrates the nutrients that contributed to each DP. Accordingly, the first DP is mainly representative of saturated, total fat, cholesterol and mono-unsaturated fatty acids (MUFA), the second DP represents minerals, vitamins and fiber, and the third DP is mainly representative of polyunsaturated fatty acids (PUFA). Additional file 2: Table S2 shows the age, gender, and race-adjusted mean of each nutrient by quartiles of the three DP.

Adjusted (by age, race, diabetes, hypertension, cholesterol, smoking and energy intake) mean of score for DP2 and DP3 were higher in men without PAD $(p<0.001)$., i.e. men without PAD had a better adherence to the diet with higher intake of minerals, vitamins and fiber (5685.0 vs. 6933.2, $p<0.001$, Table 1 ) and to the diet with higher consumption of PUFA (10.8 vs. $13.9, p<$ 0.001) compared with men with PAD. We did not find any difference for adjusted mean of each DP between women (all $p>0.05$, Table 1 ).

Furthermore, we computed adjusted (by age, race, diabetes, hypertension, cholesterol, smoking and energy 
Table 1 Adjusted mean of dietary pattern score in patients with and without peripheral artery disease (PAD)

\begin{tabular}{|c|c|c|c|c|}
\hline & & \multicolumn{2}{|l|}{ Participants } & \multirow[t]{2}{*}{$P$-value } \\
\hline & & With PAD & Without PAD & \\
\hline \multirow[t]{3}{*}{ Male } & First Dietary Pattern (Fatty Acids) & $340.1 \pm 17.5$ & $358.0 \pm 36.3$ & 0.525 \\
\hline & Second Dietary Pattern (Minerals And Vitamins) & $5679.8 \pm 221.9$ & $6959.2 \pm 441.4$ & $<0.001$ \\
\hline & Third Dietary Pattern (Poly Unsaturated Fatty Acid) & $11.2 \pm 0.6$ & $14.2 \pm 1.2$ & $<0.001$ \\
\hline \multirow[t]{3}{*}{ Female } & First Dietary Pattern (Fatty Acids) & $228 \pm 4.9$ & $252 \pm 22.33$ & 0.125 \\
\hline & Second Dietary Pattern (Minerals And Vitamins) & $4603 \pm 223.6$ & $4758 \pm 125.6$ & 0.718 \\
\hline & Third Dietary Pattern (Poly Unsaturated Fatty Acid) & $10.3 \pm 0.4$ & $10.5 \pm 1.4$ & 0.856 \\
\hline
\end{tabular}

Analysis of covariance applied to calculate age, race, diabetes, hypertension, cholesterol, smoking and energy intake adjusted mean of dietary score. Value expressed as mean \pm SEM

intake) odds for PAD for each DP stratified by gender. The results displayed that men in the highest quartile of the DP1 had a higher odds of PAD compared with the lowest quartile [odds ratio (OR): 6.32, 95\% confidence interval (95\%CI): $1.89-19.25, p<0.001]$, while men in the fourth quartile of DP2 and DP3 had lower odds for PAD compared with the first quartile (OR:0.28, 95\%CI: $0.09-0.83$, and OR: $0.44,95 \%$ CI: $0.20-0.96, p<0.001$, respectively). In women, subjects in the fourth quartile of DP2 had 81.1\% lower likelihood for PAD compared with those in the first quartile (OR: $1.66,95 \% \mathrm{CI}: 0.03-0.76, p$ $<0.001$ ). To determine the association between PAD severity (as assessed by $A B I$ value) and adhesion to each DP, adjusted (by age, race, diabetes, hypertension, cholesterol, smoking and energy intake) linear regression (with 500 bootstrap replications) was performed, reporting positive and significant associations between lower severity of PAD (higher ABI score) and higher score of DP2 and DP3 (for DP1 $=\beta$ coefficient: $0.046,95 \% \mathrm{CI}$ : $0.049-0.171$; for $\mathrm{DP} 2=\beta$ coefficient: $0.049, \quad 95 \% \mathrm{CI}$ : $0.051-0.182, p<0.001$ for both comparison).

When plasma TFAs and vitamins were adjusted for age, race, diabetes, hypertension, cholesterol, and smoking, PAD patients had significantly higher plasma concentrations of trans 9-octadecenoic acid and trans 9, trans 12-octadienoic acid and lower levels of vitamin D, retinol, retinyl stearate and retinyl palmitate $(p<0.001$ for all comparisons, Table 2).

\section{Discussion}

Our findings revealed that individuals consuming a diet with a high intake of minerals, vitamins, fiber and [second DP (DP2)] PUFA [third DP (DP3)] had a lower likelihood of PAD, while diet rich in fatty acids [first DP (DP1)]) are associated with an increased likelihood of PAD. Furthermore, individuals without PAD had higher plasma vitamins concentrations and lower plasma TFAs levels compared with PAD patients.

In line with our study, previous investigators showed that PAD diagnosis and the presence of claudication correlated with a diet rich in saturated fat, sodium, and cholesterol, and low in fiber, vitamin E, folate intake and lower levels of omega-3 PUFA [37-39]. In this regard, in a small, case-control study in an Edinburgh population, docosapentaenoic acid (PUFA) was found to be protective for PAD [6]. In patients with a history of myocardial infarction, $\alpha$-linoleic acids (ALA) as a key component of the Mediterranean diet, was shown to reduce the risk of cardiovascular events in one trial [40].

Table 2 Adjusted mean of trans fatty acids and plasma antioxidant levels in patients with and without peripheral artery disease (PAD)

\begin{tabular}{llll}
\hline & Participants & $P$-value \\
\cline { 2 - 3 } & With PAD & Without PAD & 0.842 \\
\hline Trans 9-hexadecenoic acid(uM) & $6.5 \pm 0.12$ & $6.1 \pm 0.19$ & 0.265 \\
Trans 11-octadecenoic acid (uM) & $38.1 \pm 0.36$ & $34.1 \pm 0.92$ & $<0.001$ \\
Trans 9-octadecenoic acid (uM) & $35.2 \pm 0.35$ & $30.6 \pm 0.62$ & $<0.001$ \\
Trans 9, trans 12-octadienoic acid $(\mathrm{uM})$ & $2.8 \pm 0.12$ & $2.4 \pm 0.31$ & $<0.1 \pm 0.72$ \\
Vitamin D $(\mathrm{nmol} / \mathrm{L})$ & $54.3 \pm 0.28$ & $68.4 \pm 0.64$ & $<0.001$ \\
Retinol $(\mu \mathrm{g} / \mathrm{dL})$ & $60.2 \pm 0.62$ & $0.87 \pm 0.01$ & $<0.001$ \\
Retinyl stearate $(\mu \mathrm{g} / \mathrm{dL})$ & $0.43 \pm 0.01$ & $3.0 \pm 0.10$ & $<0.001$ \\
Retinyl palmitate $(\mu \mathrm{g} / \mathrm{dL})$ & $2.1 \pm 0.28$ &
\end{tabular}

Analysis of covariance applied to calculate age, race, diabetes, hypertension, cholesterol, smoking and energy intake adjusted mean of dietary score. Value expressed as mean \pm SEM 
In a prospective study by Schiano et al., supplementing PAD patients with $2 \mathrm{~g}$ of omega-3 PUFA daily for 12 weeks led to an improvement in endothelial function relative to the non-supplemented control group, hinting at a possible slower progression of PAD [41]. With regard to potential mechanisms, it could be suggested that PUFA were shown to decrease blood triacylglycerol concentrations, inhibit the production of chemoattractants, growth factors, adhesion molecules, inflammatory eicosanoids and cytokines [38], lower blood pressure, protect against endothelial damage and atherosclerosis [39], enhance nitrite oxide production, endothelial relaxation and vascular compliance, inhibit thrombosis and cardiac arrhythmias as well as improve heart rate variability [42]. These mechanisms most probably explain the primary and secondary CVD protection afforded by PUFA consumption, suggesting that PUFA consumption may also be beneficial in PAD. In this context, the impact of PUFA on the resolution of inflammation may eventually prove to be beneficial for patients with PAD, who have a high inflammatory burden [42].

In the present study, participants with PAD had higher levels of the trans 9-octadecenoic acid and trans 9, trans 12-octadienoic acid compared with PAD-free subjects which is in line with other studies [43, 44]. Previous studies have reported that TFAs may induce endothelial dysfunction and that this may be related to an upregulation of pro-inflammatory molecules, linking vascular inflammation and thrombosis [45-47, 49]. We have recently reported the potential role of TFAs intake on inflammation in humans [48-50]. These findings are in line with the studies in animals and in vitro [51], with suggestion that in TFA-exposed blood vessels, inflammation and oxidative stress may trigger pro-thrombogenic activity of endothelial cells, which then exceeds the antithrombogenic activity [51]. Overall, TFAs may act as a risk factor of the development of PAD via their role on inflammation.

The present investigation showed that PAD patients had lower levels of vitamins. Consistently, a nationally representative cross-sectional study of adults, intakes of various nutrients, including folate and vitamins A, $\mathrm{C}$, and $\mathrm{E}$ were associated with a decreased prevalence of PAD in uncontrolled and partially controlled models [52]. Moreover, it has been demonstrated that PAD was associated with a lower consumption of vegetables, fiber, vitamins $C$ and $E$ and folate as compared with individuals without PAD [37]. The Atherosclerosis Risk In Communities (ARIC) study reported that low $25(\mathrm{OH})$ D was associated with $30 \%$ increased risk of PAD in black and white participants [53]. Moreover, in another cross-sectional analysis researchers found an $80 \%$ higher prevalence of PAD in participants in the lowest quartile of $25(\mathrm{OH}) \mathrm{D}$ compared to the highest quartile [54].

From our PCA, we have found that subjects with higher load of fiber in diet had a lower likelihood of PAD; fiber may be beneficial in reducing inflammation, as there is a strong relationship between increased fiber intake and lower hsCRP levels [55]. Furthermore, dietary fiber may have a protective effect against developing PAD, as a positive association between cereal fiber and ABI was found in the Edinburgh Artery study [4] and the Health Professional's Follow-up study [56]. Therefore, individuals with PAD and claudication may experience cardiovascular benefits by increasing dietary fiber intake, possibly via reduced inflammation and improved macro- and microvascular function.

Several mechanisms of action, have been suggested to explain the potential protective effect of fibers in PAD. Briefly, it has been hypothesized that the apparent beneficial effect of fiber intake against cardiovascular disease is mediated by cholesterol-lowering as well as reduced plasminogen activator inhibitor type 1 and factor VII activity, especially due to soluble fiber [57]. Elevated cytosolic triacylglycerols are associated with oxidative stress and can cause endothelial dysfunction [57], thus possibly predisposing to PAD development. Other mechanisms by which fiber intake might protect against PAD are: [1] increased insulin sensitivity, as fiber consumption improves insulin sensitivity by slowing the absorption of nutrients from the gut [58]; [2] reduced serum glucose levels [58]; [3] production of short chain fatty acids by gut bacteria consequently improving glucose metabolism [59], which is associated with lower LDL, blood pressure and triacylglycerols, and higher HDL [59]. Furthermore, among US adults ( $n=18,741$, mean age $=44.7$ years $)$ and after adjusting for age, sex, race, education, smoking, physical activity, BMI and total energy consumed, we observed a link between CRP, an independent risk factor for cardiovascular disease, and dietary pattern rich in fiber (unpublished data), indicating an effect of dietary fiber on the risk of cardiovascular disease mediated in part by inflammatory pathways.

The current study has several strengths. To our knowledge, it is the largest study of the association of peripheral arterial disease and plasma TFAs, antioxidants, dietary pattern with PCA methods, among a representative sample of non-institutionalized US adults. Due to a large sample size, this study is sufficiently powered to test the associations. The participant selection was based on random sampling of the general population, and therefore the results obtained from nationally representative samples are generalizable to the US population. Furthermore, detailed ABI assessments were conducted with a number of quality control procedures, including calibration of techniques prior to and annually throughout the survey. 
The findings of our study should be considered in the context of some limitations including those inherent to cross-sectional studies such as exposure-outcome temporal relationship identification and reverse causality. Moreover, we excluded 40 subjects with very high ABI $(>1.4)$ due to noncompressible arteries who are technically abnormal; such individuals should be evaluated separately in other studies with larger samples. There is also a need to assess how several factors such as ethnicity, smoker status and other characteritics can influence the intake of the nutrients considered in the present study. We need also mention that our assays sensivity for measuring Vitamin D was low.

This study has important clinical and public health implications. PAD is a chronic inflammatory condition that is associated with longstanding poor nutrition and other lifestyle habits. Understanding the interplay between vascular health status, diet and plasma levels of dietary factors (TFAs, antioxidants) is an essential and crucial step concerning any use of the findings for public health strategy and preventive actions. Future studies are needed to evaluate whether PAD patients may experience clinical benefits from improved dietary habits including regular intake of fish or daily fish oil supplements, increased dietary soluble fiber intake, consumption of adequate amounts of vitamins, and minerals, as well as reduced TFAs.

\section{Conclusion}

In conclusion, our results provide information on the association between plasma vitamins and TFAs and the likelihood of PAD. We have found that dietary factors (either via consumption or plasma concentration) are independently associated with the likelihood of PAD. The impact of the dietary items on preventing or delaying the clinical progression of PAD should be investigated in intervention trials.

\section{Additional files}

Additional file 1: Table S1. Demographic, anthropometric and clinical characteristics of participants. (DOCX $13 \mathrm{~kb}$ )

Additional file 2: Table S2. Age, Gender, and Race Adjusted Mean of Nutrient Intakes Across Quartiles of Each Dietary Pattern. (DOCX 22 kb)

\section{Acknowledgments}

MM was supported by a TWAS and Chinese Academy of Sciences, during the preparation of this manuscript.

\section{Funding}

None

Availability of data and materials

all the data are from public access database.

\section{Authors' contributions}

MM: Study conception and design, Analysis and interpretation of data. Drafting of manuscript. NDW, NK, DPM, and MB: Critical revision and interpretation of data. All authors read and approved the final manuscript.

\section{Ethics approval and consent to participate}

for the data collection and physical examination of the NHANES, informed consent was obtained from all adult participants, and the National Centre for Health Statistics Research Ethics Review Board approved the protocol.

\section{Consent for publication}

for the data collection and physical examination of the NHANES, informed consent (publication) was obtained from all adult participants, and the National Centre for Health Statistics Research Ethics Review Board approved the protocol.

\section{Competing interests}

The corresponding author is responsible for submitting a competing financial interest statement on behalf of all authors of the paper. The authors declare no competing financial interests.

\section{Publisher's Note}

Springer Nature remains neutral with regard to jurisdictional claims in published maps and institutional affiliations.

\section{Author details}

'Key State Laboratory of Molecular Developmental Biology, Institute of Genetics and Developmental Biology, Chinese Academy of Sciences, Chaoyang, Beijing, China. ${ }^{2}$ Institute of Genetics and Developmental Biology. International College, University of Chinese Academy of Science (IC-UCAS), West Beichen Road, Chaoyang, China. ${ }^{3}$ Heart Disease Prevention Program, Division of Cardiology, University of California, Irvine, CA, USA. ${ }^{4}$ Second Propedeutic Department of Internal Medicine, Medical School, Aristotle University of Thessaloniki, Hippokration Hospital, Thessaloniki, Greece. ${ }^{5}$ Department of Clinical Biochemistry, Royal Free Campus, University College London Medical School, University College London (UCL), London, UK. ${ }^{6}$ Department of Hypertension, Chair of Nephrology and Hypertension, Medical University of Lodz, Lodz, Poland. PPolish Mother's Memorial Hospital Research Institute (PMMHRI), Lodz, Poland.

Received: 19 September 2017 Accepted: 4 December 2017

Published online: 28 December 2017

\section{References}

1. Brevetti G, Giugliano G, Brevetti L, Hiatt WR. Inflammation in peripheral artery disease. Circulation. 2010;122(18):1862-75.

2. Lewis CD. Peripheral arterial disease of the lower extremity. J Cardiovasc Nurs. 2001;15(4):45-63. quiz 96-7

3. Lane JS, Magno CP, Lane KT, Chan T, Hoyt DB, Greenfield S. Nutrition impacts the prevalence of peripheral arterial disease in the United States. J Vasc Surg. 2008;48(4):897-904.

4. Donnan PT, Thomson M, Fowkes FG, Prescott RJ, Housley E. Diet as a risk factor for peripheral arterial disease in the general population: the Edinburgh artery study. Am J Clin Nutr. 1993;57(6):917-21.

5. Gimeno SG, Hirai AT, Harima HA, Kikuchi MY, Simony RF, de Barros N Jr, et al. Fat and fiber consumption are associated with peripheral arterial disease in a cross-sectional study of a Japanese-Brazilian population. Circ J. 2008;72(1):44-50.

6. Leng GC, Horrobin DF, Fowkes FG, Smith FB, Lowe GD, Donnan PT, et al. Plasma essential fatty acids, cigarette smoking, and dietary antioxidants in peripheral arterial disease. A population-based case-control study. Arterioscler Thromb. 1994;14(3):471-8.

7. Langlois M, Duprez D, Delanghe J, De Buyzere M, Clement DL. Serum vitamin C concentration is low in peripheral arterial disease and is associated with inflammation and severity of atherosclerosis. Circulation. 2001:103(14):1863-8

8. Schiano V, Laurenzano E, Brevetti G, De Maio Jl, Lanero S, Scopacasa F, Chiariello M. Omega-3 polyunsaturated fatty acid in peripheral arterial disease: effect on lipid pattern, disease severity, inflammation profile, and endothelial function. Clin Nutr. 2008(2):241-7. doi:10.1016/j.clnu.2007.11.007. Epub 2008 Jan 31 
9. Hu FB. Dietary pattern analysis: a new direction in nutritional epidemiology. Curr Opin Lipidol. 2002;13(1):3-9.

10. Newby P, Tucker KL. Empirically derived eating patterns using factor or cluster analysis: a review. Nutr Rev. 2004;62(5):177-203.

11. Zuo H, Shi Z, Yuan B, Dai Y, Pan X, Wu G, et al. Dietary patterns are associated with insulin resistance in Chinese adults without known diabetes. Br J Nutr. 2013;109(09):1662-9.

12. Kant AK. Dietary patterns and health outcomes. J Am Diet Assoc. 2004; 104(4):615-35.

13. Mazidi M, Pennathur S, Afshinnia F. Link of dietary patterns with metabolic syndrome: analysis of the National Health and Nutrition Examination Survey. Nutr Diabetes. 2017;7(3):e255. doi:10.1038/nutd.2017.11.

14. Mazidi M, Kengne AP. Nutrient patterns and their relationship with general and central obesity in US adults. Eur J Clin Invest. 2017 doi:10.1111/eci. 12745. [Epub ahead of print].

15. Slattery ML. Defining dietary consumption: is the sum greater than its parts? Am J Clin Nutr. 2008:88(1):14-5.

16. Mazidi M, Rezaie P, Ferns GA, Gao HK. Impact of different types of tree nut, peanut, and soy nut consumption on serum C-reactive protein (CRP): a systematic review and meta-analysis of randomized controlled clinical trials. Medicine (Baltimore). 2016:95(44):e5165.

17. Klein-Platat C, Drai J, Oujaa M, Schlienger JL, Simon C. Plasma fatty acid composition is associated with the metabolic syndrome and low-grade inflammation in overweight adolescents. Am J Clin Nutr. 2005;82(6): 1178-84.

18. https://www.cdc.gov/nchs/index.htm. [accessed 19.08.13].

19. Ahluwalia N, Andreeva VA, Kesse-Guyot E, Hercberg S. Dietary patterns, inflammation and the metabolic syndrome. Diabetes Metab. 2013;39(2): 99-110.

20. Ahluwalia N, Dwyer J, Terry A, Moshfegh A, Johnson C. Update on NHANES dietary data: focus on collection, release, analytical considerations, and uses to inform public policy. Adv Nutr. 2016;7(1):121-34.

21. Mazidi M, Kengne AP, Banach M. Mineral and vitamins consumption is associated with longer telomeres among US adults. Pol Arch Med Wewn. 2017;127(2):87-90. doi:10.20452/pamw.3927.

22. Moshfegh AJ, Rhodes DG, Baer DJ, Murayi T, Clemens JC, Rumpler WV, et al. The US Department of Agriculture Automated Multiple-Pass Method reduces bias in the collection of energy intakes. Am J Clin Nutr. 2008;88(2): 324-32.

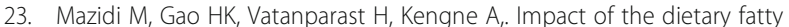
acid intake on C-reactive protein levels in US adults. Medicine (Baltimore). 2017;96(7):e5736. doi:10.1097/MD.0000000000005736.

24. Mazidi M, Toth PP, Banach M. C-reactive Protein Is Associated With Prevalence of the Metabolic Syndrome, Hypertension, and Diabetes Mellitus in US Adults. Angiology. 2017;1:3319717729288. doi:10.1177/ 0003319717729288. [Epub ahead of print].

25. Mohsen Mazidi EDM. Maciej Banach. The association of telomere length and serum 25-hydroxyvitamin D levels in US adults: the National Health and nutrition examination survey. Arch Med Sci. 2017;13(1):61-5.

26. Needham BL, Adler N, Gregorich S, Rehkopf D, Lin J, Blackburn EH, et al. Socioeconomic status, health behavior, and leukocyte telomere length in the National Health and Nutrition Examination Survey, 1999-2002. Social science \& medicine (1982). 2013:85:1-8.

27. Remer T. Influence of nutrition on acid-base balance-metabolic aspects. Eur J Nutr. 2001:40(5):214-20.

28. National Center for Health Statistics CfDCaPNHaNESA, https://www.cdc.gov/ nchs/index.htm f. Accessed 4 Feb 2017.

29. Teegala SM, Willett WC, Mozaffarian D. Consumption and health effects of trans fatty acids: a review. J AOAC Int. 2009;92(5):1250-7.

30. Risé P, Eligini S, Ghezzi S, Colli S, Galli C. Fatty acid composition of plasma, blood cells and whole blood: relevance for the assessment of the fatty acid status in humans. Prostaglandins Leukot Essent Fat Acids. 2007;76(6):363-9.

31. Nutritional. Biochemistry Branch DoLS, National Center for Environmental Health (n.d.) Laboratory ProcedureManual 25-Hydroxyvitamin D.

32. Zhang $Y$, Huang J, Wang P. A prediction model for the peripheral arterial disease using NHANES data. Medicine (Baltimore). 2016;95(16):e3454. doi:10.1097/MD.0000000000003454.

33. Statistics. NCFH. ANALYTIC AND REPORTING GUIDELINES https://www.cdc. gov/nchs/index.htm nhanes 03 04/nhanes analytic guidelines dec 2005.pdf. Accessed 4 Feb 2017.
34. Khayyatzadeh SS, Moohebati M, Mazidi M, Avan A, Tayefi M, Parizadeh SM, et al. Nutrient patterns and their relationship to metabolic syndrome in Iranian adults. Eur J Clin Investig. 2016;46(10):840-52.

35. Stanhope KL. Role of fructose-containing sugars in the epidemics of obesity and metabolic syndrome. Annu Rev Med. 2012;63:329-43.

36. W W. Nutritional epidemiology. Oxford University Press, Oxford. 2013.

37. Antonelli-Incalzi R, Pedone C, McDermott MM, Bandinelli S, Miniati B, Lova RM, et al. Association between nutrient intake and peripheral artery disease: results from the InCHIANTI study. Atherosclerosis. 2006; 186(1):200-6.

38. Grenon SM, Conte MS, Nosova E, Alley H, Chong K, Harris WS, et al. Association between $n-3$ polyunsaturated fatty acid content of red blood cells and inflammatory biomarkers in patients with peripheral artery disease. J Vasc Surg. 2013;58(5):1283-90.

39. Holy EW, Forestier M, Richter EK, Akhmedov A, Leiber F, Camici GG, et al. Dietary alpha-linolenic acid inhibits arterial thrombus formation, tissue factor expression, and platelet activation. Arterioscler Thromb Vasc Biol. 2011;31(8): $1772-80$.

40. de Lorgeril M, Salen P, Martin JL, Monjaud I, Delaye J, Mamelle N. Mediterranean diet, traditional risk factors, and the rate of cardiovascular complications after myocardial infarction: final report of the Lyon diet heart study. Circulation. 1999;99(6):779-85.

41. Schiano V, Laurenzano E, Brevetti G, De Maio Jl, Lanero S, Scopacasa F, et al. Omega-3 polyunsaturated fatty acid in peripheral arterial disease: effect on lipid pattern, disease severity, inflammation profile, and endothelial function. Clin Nutr. 2008;27(2):241-7.

42. Grenon SM, Aguado-Zuniga J, Hatton JP, Owens CD, Conte MS, HughesFulford M. Effects of fatty acids on endothelial cells: inflammation and monocyte adhesion. J Surg Res. 2012;177(1):e35-43.

43. Benatar JR, Gladding P, White HD, Zeng I, Stewart RA. Trans-fatty acids in New Zealand patients with coronary artery disease. Eur I Cardiovasc Prev Rehabil. 2011:18(4):615-20.

44. Siri-Tarino PW, Chiu S, Bergeron N, Krauss RM. Saturated fats versus polyunsaturated fats versus carbohydrates for cardiovascular disease prevention and treatment. Annu Rev Nutr. 2015;35:517-43.

45. Harvey KA, Walker $\mathrm{CL}, \mathrm{Xu} Z$ Z, Whitley P, Siddiqui RA. Trans fatty acids: induction of a pro-inflammatory phenotype in endothelial cells. Lipids. 2012 47(7):647-57.

46. Lopez-Garcia E, Schulze MB, Meigs JB, Manson JE, Rifai N, Stampfer MJ, et al. Consumption of trans fatty acids is related to plasma biomarkers of inflammation and endothelial dysfunction. J Nutr. 2005;135(3):562-6.

47. Petaja J. Inflammation and coagulation. An overview. Thromb Res. 2011;127 Suppl 2:S34-7.

48. Mazidi M, Shivappa N, Wirth MD, Hebert JR, Vatanparast H, Kengne AP. The association between dietary inflammatory properties and bone mineral density and risk of fracture in US adults. Eur J Clin Nutr. 2017;71(11):12731277. doi:10.1038/ejcn.2017.133.

49. Mazidi M, Gao HK, Shivappa N, Wirth MD, Hebert JR, Kengne AP. The relationship of plasma trans fatty acids with dietary inflammatory index among US adults. Lipids Health Dis. 2017;16(1):147. doi:10.1186/s12944017-0527-1.

50. Mazidi M, Gao HK, Kengne AP. Markers Are Positively Associated with Serum trans-Fatty Acids in an Adult American Population. J Nutr Metab. 2017;2017: 3848201. doi:10.1155/2017/3848201. Epub 2017 Jul 11.

51. Kondo K, Ishida T, Yasuda T, Nakajima H, Mori K, Tanaka N, et al. Trans-fatty acid promotes thrombus formation in mice by aggravating antithrombogenic endothelial functions via toll-like receptors. Mol Nutr Food Res. 2015;59(4):729-40.

52. Naqvi AZ, Davis RB, Mukamal KJ. Nutrient intake and peripheral artery disease in adults: key considerations in cross-sectional studies. Clin Nutr. 2014;33(3):443-7.

53. Rapson IR, Michos ED, Alonso A, Hirsch AT, Matsushita K, Reis JP, et al. Serum 25 -hydroxyvitamin $D$ is associated with incident peripheral artery disease among white and black adults in the ARIC study cohort. Atherosclerosis. 2017;257:123-9.

54. Melamed ML, Muntner P, Michos ED, Uribarri J, Weber C, Sharma J, et al. Serum 25-hydroxyvitamin D levels and the prevalence of peripheral arterial disease: results from NHANES 2001 to 2004. Arterioscler Thromb Vasc Biol. 2008;28(6):1179-85.

55. King DE, Egan BM, Geesey ME. Relation of dietary fat and fiber to elevation of C-reactive protein. Am J Cardiol. 2003;92(11):1335-9. 
56. Merchant AT, Hu FB, Spiegelman D, Willett WC, Rimm EB, Ascherio A. Dietary fiber reduces peripheral arterial disease risk in men. J Nutr. 2003; 133(11):3658-63.

57. Hunninghake DB, Miller VT, LaRosa JC, Kinosian B, Brown V, Howard WJ, et al. Hypocholesterolemic effects of a dietary fiber supplement. Am J Clin Nutr. 1994;59(5):1050-4.

58. Mazidi M, Kengne AP, Mikhailidis DP, Toth PP, Ray KK, Banach M. Dietary food patterns and glucose/insulin homeostasis: a cross-sectional study involving 24,182 adult Americans. Lipids Health Dis. 2017;16(1):192.

59. Mazidi M, Rezaie P, Kengne AP, Mobarhan MG, Ferns GA. Gut microbiome and metabolic syndrome. Diabetes Metab Syndr. 2016;10(2 Suppl 1):S150-7.

Submit your next manuscript to BioMed Central and we will help you at every step:

- We accept pre-submission inquiries

- Our selector tool helps you to find the most relevant journal

- We provide round the clock customer support

- Convenient online submission

- Thorough peer review

- Inclusion in PubMed and all major indexing services

- Maximum visibility for your research

Submit your manuscript at www.biomedcentral.com/submit 